\title{
The Impact of Web-Based Middleware Systems on Training and Assessment through In-House Develop System
}

\author{
Johnson Dehinbo \\ Tshwane University of Technology, Pretoria, South Africa
}

Dehinbo.j@tng.ac.za

\begin{abstract}
This study is aimed at determining the impact that web-based middleware systems can imprint in enhancing learning and assessment. Representative samples of students attending introductory computer classes at the Technikon, were assessed to determine the level of their knowledge and use of the Internet. Very few of them demonstrate high Internet knowledge and awareness. Over $80 \%$ indicate willingness to surf the web more, if there is a compelling situation that forces or encourages them. One group of students, were given assignments and tests throughout the semester via the on-line system developed, and were subsequently reassessed at the end of the semester. The result showed dramatic improvement in the level of their knowledge and use of the Internet as well as increased speed of carrying out assigned tasks on the Internet. The study concludes there is a huge impact that web-based middleware can imprint in enhancing learning even in contact institutions.
\end{abstract}

Keywords: Web-based, middleware, Internet, programming, applications

\section{Introduction}

The society today is in an era where valuable information spread all over the world can easily be obtained at the click of a button. The advent of the Internet and the World Wide Web has made this goal realistic. Valuable information can be stored on a server and a web page can be written to point to this information. Interested users can use their browsers to connect to and browse this page, which is transferred and executed or displayed on the users' (or clients') computer. Clicking on the hypertext representing the desired application system on this page, connects to the system using the URL (Uniform Resource Locator) for the database or system. The introduction to your paper goes in this section. Users can then download this information to their disk. The web pages are written using Hyper Text Markup Language (HTML). Recent technologies involve integrating web pages with programming languages such as Java, leading to effective web-based applications. These new developments are permeating into educational systems. It therefore becomes imperative to examine the impact of such technologies in improving the quality of training and assessment.

Material published as part of these proceedings, either on-line or in print, is copyrighted by Informing Science. Permission to make digital or paper copy of part or all of these works for personal or classroom use is granted without fee provided that the copies are not made or distributed for profit or commercial advantage AND that copies 1) bear this notice in full and 2) give the full citation on the first page. It is permissible to abstract these works so long as credit is given. To copy in all other cases or to republish or to post on a server or to redistribute to lists requires specific permission from the publisher at Publisher@InformingScience.org
This research was necessitated due to perceived lack of enthusiasm on the part of TNG students to maximize the use of Internet as a source of diverse wealth of information. Also, there is the need to maximize lecturing time and reduce paper costs by making lec- 
tures, assignments and tests available on the web.

\section{Literature Review}

The interdisciplinary nature of this research requires both background and in depth knowledge of specific areas related to the problem being investigated.

\section{Traditional Modes of Training and Assessment}

From times immemorial, the traditional modes of training and assessment include oral, written (paper-based) and recently the computer-based off-line written forms. The major limitations of these systems include the need for contact between the trainer/assessor and trainee/assessee, lack of time flexibility, and the cost of storage of training and assessment materials.

\section{Web-based Modes of Training and Assessment}

Web-based modes of training and assessment seem to be striving to eliminate the limitations of traditional modes of training and assessment. Designing such web-based applications requires the knowledge of programming languages such as Java (Java servlets and JavaServer Pages), Visual Basic (Active Server Pages using VBScript), Personal Home Page (PHP) as well as knowledge of the HyperText Markup Language (HTML). This study involves the implementation using the HTML in conjuction with Java.

\section{Application Models}

The two application models commonly used are the two-tier and the three-tier application models:

In the two-tier model, a Java applet or application talks directly to the database or flat file. This is referred to as a client/server configuration, with the user's machine as the client, and the machine housing the database / file as the server. This approach has the benefit of moving front-end processing off the server (Taylor, 1997).

The three-tiered Java Applications is the server interface approach. In the three-tier model, commands are sent to a "middle tier" of services, which then send SQL statements to the database. The database processes the SQL statements and sends the results back to the middle tier, which then sends them to the user.

This study uses the client-server model and examines how to construct a communication path between the web browser in a client, web server, servlets written in Java and the database through JDBC or a flat text file. The study ends with the implementation of the school assignment system whereby students can connect on-line for the collection, answering and submission of assignments through the World Wide Web.

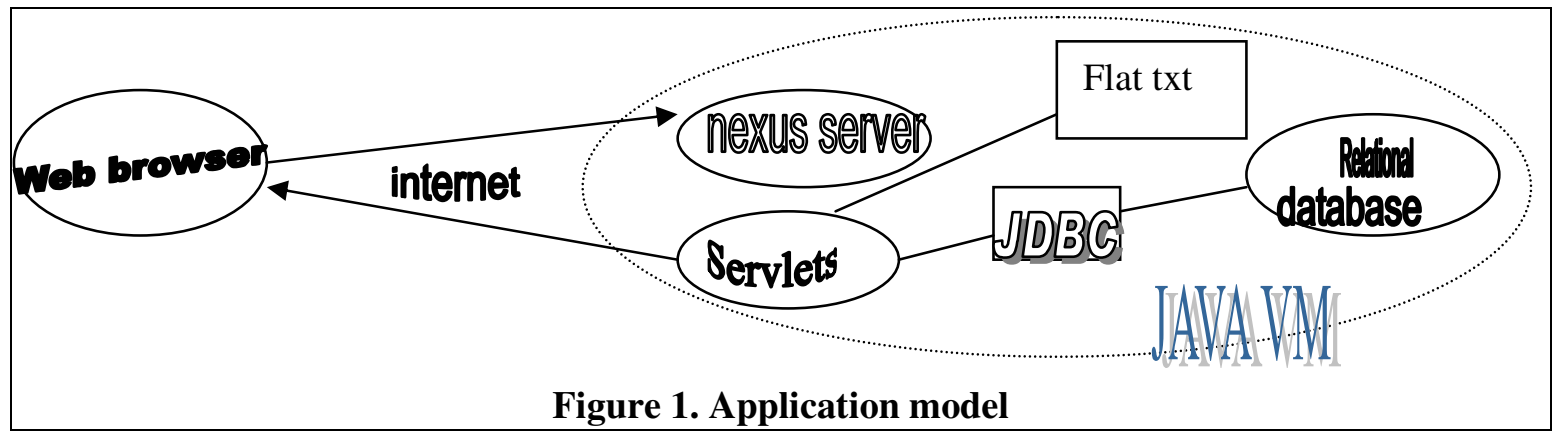


Various programming and scripting languages can be used to implement the model above. These include Java Servlets, Java Server Pages, Active Server Pages and PHP. (See Figure 1.)

\section{Why Use Java?}

Java provides a number of important features that make it the language of choice for enterprise applications. The language is portable and object-oriented. It provides a rich runtime environment that creates a security layer between the application and the client machine.

The Java application runs in an environment known as the Java virtual machine, which consists of an interpreter that reads and interprets the bytecodes that make up the application. The interpreter dynamically loads classes when they are needed. Additional portions of the virtual machine perform garbage collection, freeing memory when it is no longer needed, and managing other resources. Additional runtime features check for array bounds violations and null pointers (Sun Microsystems, 1999).

From my own experience, I observed that a very important feature is the integration of Java to web pages through HTML. In a brief summary, HTML makes it possible to describe how static Web pages should appear, including tables and pictures. With cgi-bin, it is also possible to have a limited amount of two-way interaction (forms etc). However, rapid interaction with Web pages written in HTML is not possible. To make it possible to have highly interactive Web pages, a different mechanism is needed. One such mechanism is the Java ${ }^{\mathrm{TM}}$ language and interpreter.

The Java language also supports the Servlet API. The Java servlet API is a standard for extending HTTP servers and other connection-oriented servers. It allows generation of dynamic content from Java objects executing in the servers virtual machine.

The use of servlets also offers some advantages over cgi-bin programs. These includes servlets surviving across invocations and thus simplifies creation of services which has to maintain state, as well as enterprise computing in which using Java in the backend means one can leverage Java technology such as Java ORBs, JDBC etc.

\section{Web Servers and Servlets}

In the two-tier model, Web servers and servlets act as the "second tier" of services, to which browsers communicate, and which executes the java codes, and sends the results back to the user. Only the user requests are communicated to the server and the results of operations communicated back to the client as HTML pages. This improves performance as all processing operations are done on the server, and the amount of information passed between the server and the client is also reduced as bytecodes are not downloaded to the client machine as in applet, but mere HTML output pages are passed (Sun Microsystems, 1999).

According to Cooper (2001), Servlets are managed by a Servlet container, which is a component of any web server capable of running Servlets. Servlets manage Internet connections by a method called "service" which has two parameters - a request object and a response object. These receive and respond to requests from clients respectively.

More specifically, a Servlet class is created by extending the GenericServlet class or the HTTPServlet class of the Java SDK API. In this case, HTTP servlets will usually inherit from class HttpServlet and in particular they will implement:

\section{Servlet.service (HttpServletRequest, HttpServletResponse)}

which is the method invoked by the server in response to HTTP requests, and which is responsible for generating an HTTP response (Magelang Institute, 1998). In a request/response model, a 
client sends a request message to a server and the server responds by sending back a reply message.

The most important methods of the HTTPServlet class are the two service-methods doGET and doPOST. These respond to GET and POST method connections respectively. Usually, they are over-ridden with identical code. Each has request and response parameters (Cooper, 2001).

As adapted from Sun Educational services (2002), Java Servlet programs have the following advantages:

Each request is run in a separate thread, so Servlet request processing is significantly faster than traditional CGI processing.

$>$ Servlets are scalable. Many more requests can be executed because the Web container uses a thread rather than an operating system process, which is a limited system resource.

$>$ Servlets are robust and object-oriented. One has all the capabilities of the Java programming language when writing the programs.

$>$ Servlets can only be written in the Java programming language, which makes them easy to write if you know the Java programming language. However, using Servlets to generate pages with dynamic content requires application development expertise.

$>$ Servlets are platform independent, because they are written in the Java programming language.

$>$ Servlets have access to logging capabilities. Most CGI programs do not.

$>$ The Web container provides additional services to the Servlets, such as error handling and security.

As adapted from Sun Educational services (2002), Servlets also have the following disadvantages:

$>$ Servlets often contain both business logic and presentation logic. Presentation logic is anything that controls how the application presents information to the user. Generating the HTML response within the Servlet code is presentation logic. Business logic is anything that manipulates data in order to accomplish something, such as storing data. Mixing both business logic and presentation logic means that whenever a Web page changes, the Servlet must be re-written, recompiled, and redeployed.

$>$ Servlets must handle concurrency issues.

The disadvantage of mixing both business logic and presentation logic led to the development of the Model 2 architecture which involved combining with template pages, including the JavaServer Pages technology (Sun Educational services, 2002).

\section{Methodology}

\section{Research Design}

The approach that was used is a survey design. The questionnaires contain closed questions. Questionnaires were administered to respondents using the Internet lab at the technikon. This is necessary in order to solicit information from a range of users of these labs.

\section{Population}

The intended population is the total of all students using the Internet lab at the technikon. These are students registered for the introductory computer courses namely; End user Computing, Principles of Information Systems and Information Systems. For about 75 students per class, these total to about 225 students. 


\section{Sampling and Assessment}

According to Corbetta (2003:216), for a 95\% confidence level, a population of 100 requires a sample size of 80 while a population of 300 requires a sample size of 170 . Therefore a sample size of about 100-125 would suffice for our population size of 225. Random representative samples of 50 students each from the various groups of TNG students mentioned above were assessed to determine the level of their knowledge and use of the Internet. They were initially assessed using questionnaires, and then they were given some tasks to do on the Internet. Such tasks include subscribing for an email account on free web-based email services such as yahoo or hotmail.

\section{Data Collection}

A total of 150 questionnaires were distributed. In all, a total of 120 questionnaires were completed and returned.

\section{Hypothesis}

The null hypothesis is that changing to an on-line test may not necessarily improve the Internet knowledge of the students.

\section{Assumptions}

It is assumed that respondents have all done the theoretical component of the subject and that they have acquired necessary background knowledge of using computers in the first 2 months of the semester.

\section{Limitations}

This study is limited to respondents doing preparatory courses in computing. It does not apply to students studying Computer science and Information Systems as their majors. This is because these would definitely have acquired various knowledges in other subjects that would enhance their knowledge of the Internet.

\section{Discussion of the Results}

\section{Internet Awareness}

From the total number of 120 responses received, most of the respondents are aware of the Internet as a very important tool in education, learning and future endeavors. The result is presented in the table 1 given below showing \% agreeing to the assertions:

Table 1. Percentage of Respondents agreeing to the Internet awareness assertions

\begin{tabular}{|l|c|}
\hline \multicolumn{1}{|c|}{ Assertions } & $\%$ \\
\hline $\begin{array}{l}\text { The Internet is a very important means of obtaining various types of informa- } \\
\text { tion }\end{array}$ & 89 \\
\hline $\begin{array}{l}\text { The world wide web consist of various web sites of organisations ranging from } \\
\text { schools, banks, supermarkets to government organizations }\end{array}$ & 92 \\
\hline $\begin{array}{l}\text { On-line shopping, e-learning, e-banking and newsgroups are conducted over } \\
\text { the Internet }\end{array}$ & 86 \\
\hline
\end{tabular}




\begin{tabular}{|l|c|}
\hline $\begin{array}{l}\text { The various free web-based email facilities on the Internet such as yahoo, } \\
\text { hotmail enhance communication even among previously disadvantaged } \\
\text { people }\end{array}$ & 95 \\
\hline Communications through the Internet is necessary for this new Generation & 97 \\
\hline $\begin{array}{l}\text { Internet will be a valuable tool for me when I start looking for inservice } \\
\text { training and jobs }\end{array}$ & 89 \\
\hline $\begin{array}{l}\text { I am looking forward to days when I will be conducting various services on } \\
\text { the Internet }\end{array}$ & 92 \\
\hline
\end{tabular}

\section{Internet Knowledge}

Very few of the respondents demonstrate high Internet knowledge. The result about their Internet knowledge is given Table 2 .

Futhermore, while over 95\% have used either Microsoft Word or Excel, less than 20\% have used either Internet explorer or Netscape Navigator. About $70 \%$ have never accessed the Internet while about $18 \%$ access the Internet weekly or monthly. None of the respondents access the Internet every day. Only $5 \%$ can explicitly mention some reasons why some web addresses always return some errors and $8 \%$ demonstrated ability to correct minor errors on email / web addresses.

Interestingly, over $80 \%$ indicate willingness to surf the web more, if there is a compelling situation that forces or encourages them. They think doing your assignments over the Internet will encourage them to become more versatile on the Internet? This led to the recommendation to develop an on-line assignment and testing software.

The online assignment and testing software is therefore expected to propel students to work on the Internet. While a student is answering the assignments on the web, there is a great likelihood of getting more familiar with the use of the Internet as source of valuable information.

Table 2. Percentage of Respondents having Internet knowledge

\begin{tabular}{|l|l|l|l|}
\hline \multicolumn{1}{|c|}{ Assertions } & Yes & No & $\begin{array}{l}\text { Don't } \\
\text { know }\end{array}$ \\
\hline $\begin{array}{l}\text { Have been using the Internet before registering for this } \\
\text { subject? }\end{array}$ & 29 & 70 & 1 \\
\hline $\begin{array}{l}\text { Have been using the Internet effectively since they started } \\
\text { the practicals for this subject? }\end{array}$ & 56 & 44 & 0 \\
\hline $\begin{array}{l}\text { Do you think the knowledge of Internet and world wide } \\
\text { web will add value to you in your future endeavors? }\end{array}$ & 87 & 20 & 3 \\
\hline Knowledge of how to start browsing the Internet? & 55 & 44 & 1 \\
\hline Possession of an email address? & 52 & 48 & 0 \\
\hline $\begin{array}{l}\text { Knowledge of the address of web sites where you can reg- } \\
\text { ister for free email account? }\end{array}$ & 45 & 51 & 4 \\
\hline Knowledge of how to register for free email account? & 40 & 58 & 1 \\
\hline $\begin{array}{l}\text { Knowledge of the address of web sites where you can do } \\
\text { some shopping online? }\end{array}$ & 27 & 69 & 4 \\
\hline $\begin{array}{l}\text { Knowledge of the addresses of web sites where you can } \\
\text { do banking operations? }\end{array}$ & 32 & 68 & 0 \\
\hline Having Internet facility at home? & 5 & 92 & 3 \\
\hline Going to Internet cafes to use their services? & 7 & 92 & 1 \\
\hline $\begin{array}{l}\text { Knowledge of how to search for a particular information } \\
\text { on the Internet? }\end{array}$ & 11 & 85 & 4 \\
\hline
\end{tabular}




\section{Software Development}

\section{Application System Design}

The software development involved setting up web server and programming using Java and HTML. Students' assignments are converted to HTML format which is embedded in Java Servlets/programs which are powered by an Apache Tomcat Server, thus becoming assessable via web browsers. Students can then fill-in answers and on clicking submit, the servlets ensured onward transmission to the lecturer's computer. Below is a brief methodology for the software development:

The Java 2 Servlet Development kit and the Tomcat Servlet / JSP engine are downloaded, installed and configured. The configuration involved setting the PATH statement to include the Java executables, and then setting the CLASSPATH environment to include the Tomcat library files serving as Servlet add-ons to the Java run-time executables.

Web pages (.html) are then designed along with JSP files which are loaded as users connect to the site address.

These modules connect to the server and display the assignment in the form of an HTML FORM on the client's screen. The client can then fill-in his or her name and student number, as well as the answers to the questions online. There are different types of questions represented viz;

$\checkmark$ Multiple choice

$\checkmark$ Mix and Match

$\checkmark$ Fill-in the missing words

$\checkmark$ Definitions and explanations

These are shown in Figure 2 (a-d).

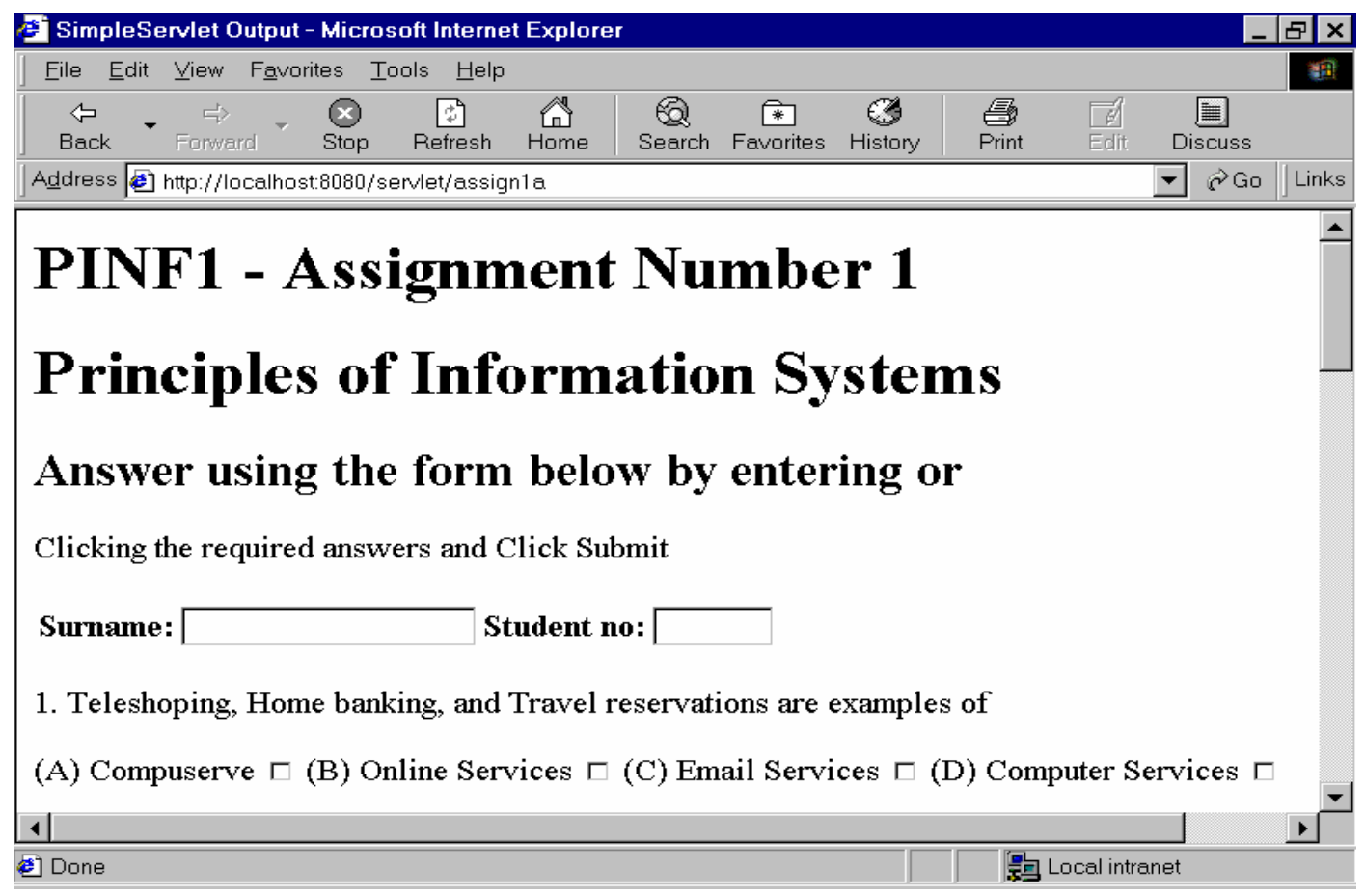

Figure 2a. Online assignment form's beginning 


\begin{tabular}{|c|c|c|c|c|c|c|c|c|c|c|c|c|}
\hline File & Eqdit & View & Favorites & Iools 브elp & & & & & & & & 再 \\
\hline $\begin{array}{r}\text { • } \\
\text { Back }\end{array}$ & $\checkmark$ & $\begin{array}{c}\Rightarrow \\
\text { Fonward }\end{array}$ & $\underset{\text { Stop }}{\boldsymbol{x}}$ & Refresh & $\begin{array}{l}\text { 全 } \\
\text { Home }\end{array}$ & $\begin{array}{l}\text { Ga } \\
\text { Search }\end{array}$ & $\begin{array}{c}\text { * } \\
\text { Favorites }\end{array}$ & $\begin{array}{l}63 \\
\text { History }\end{array}$ & $\begin{array}{c}\underset{9}{G} \\
\text { Print }\end{array}$ & $\frac{E d}{E d i t}$ & Discuss & \\
\hline Address & 5 & http://loc & calhost:8080, & /servlet/assig & & & & & & & $\nabla$ लGo & Links \\
\hline
\end{tabular}

2. A record contains a set of related $?$

(A) Files $\circ$ (B) Fields $\odot$ (C) Databases $\circ$ (D) Keys $\odot$

(A) Point to People Protocol

(B) Pole to People Protocol

3. PPP means: (Scroll \& click from A-D) (C) Point to Point Protoco

4. WWW is accessed by special software called

5. Define Database

Click the button for one that is perfectly related or perfectly unrelated

$$
4 \mid
$$

\section{Figure 2b. Online assignment form's continuation}

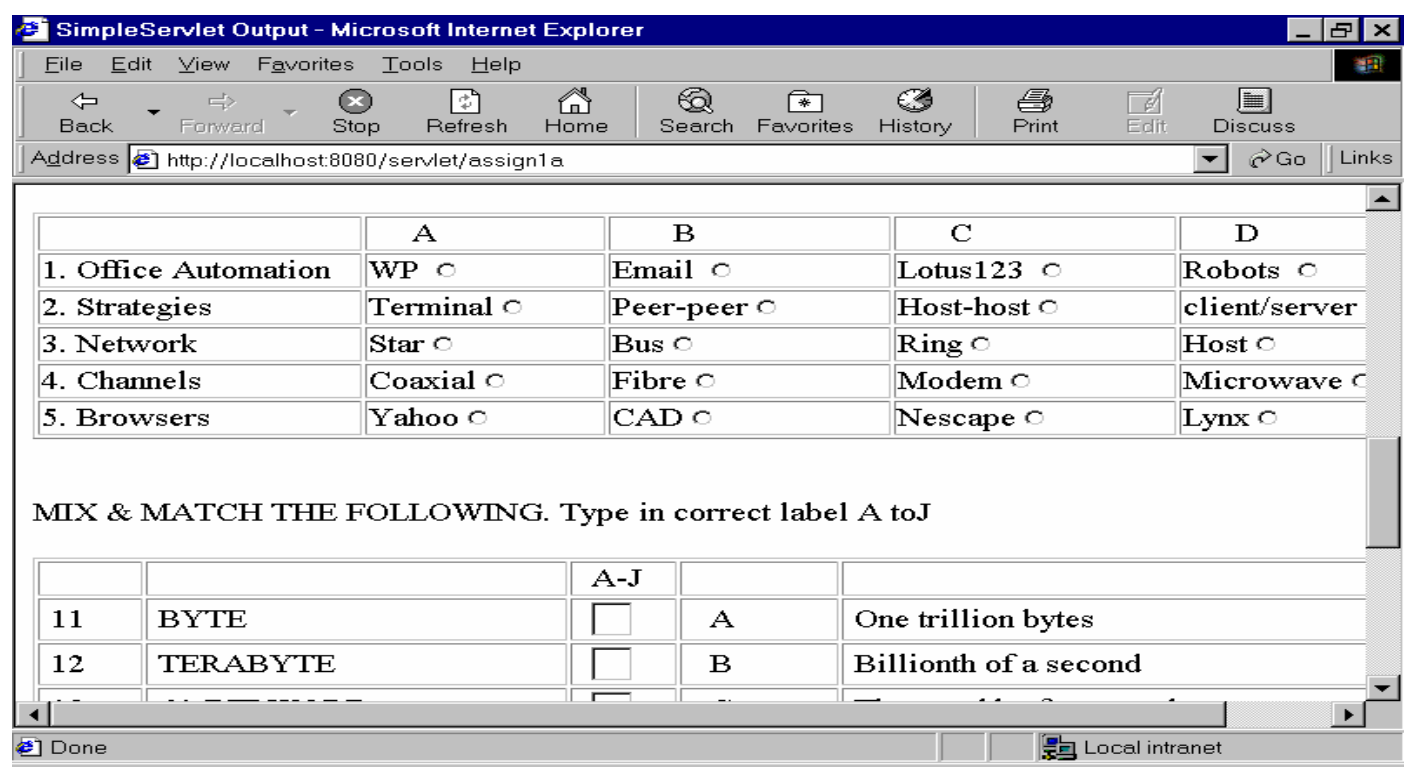

Figure 2c. Online assignment form's continuation 


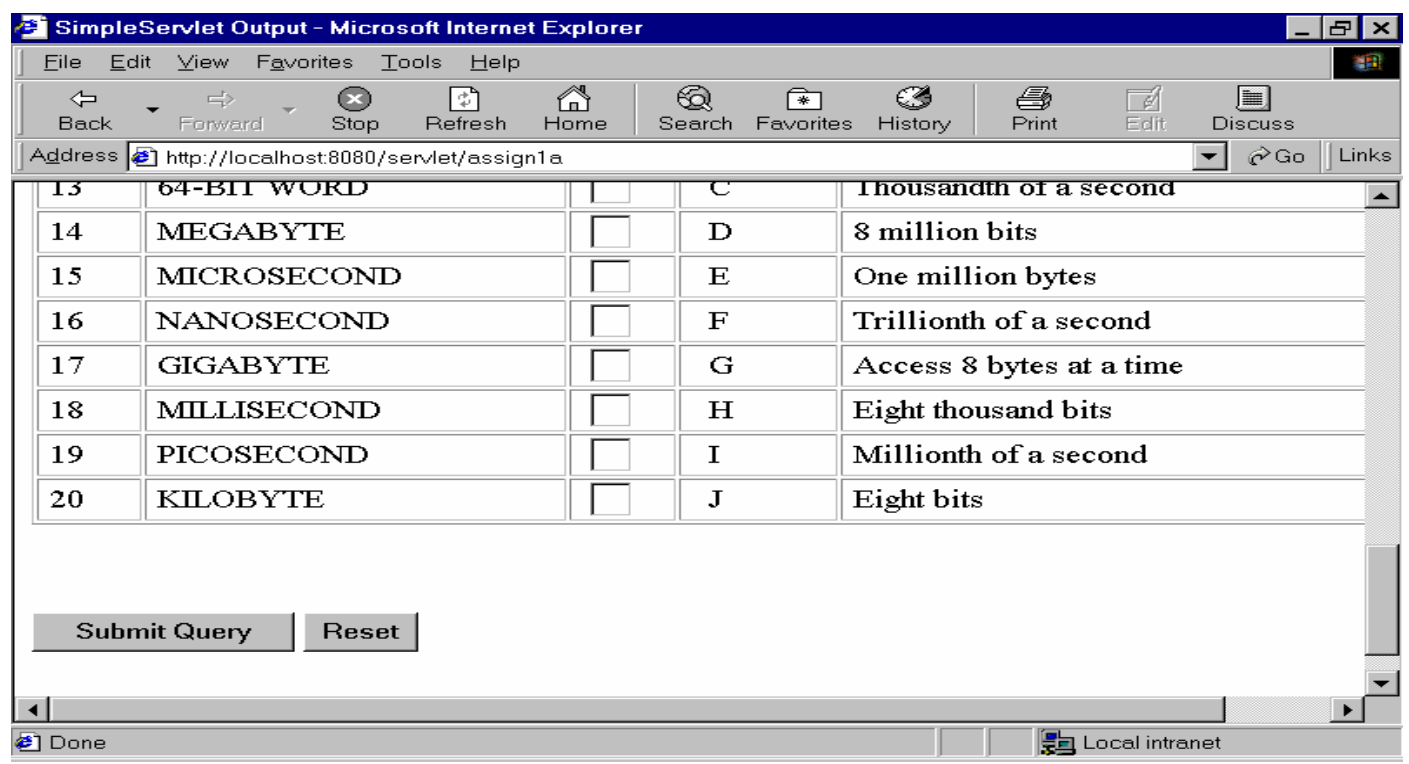

Figure 2d. Online assignment form's submission page

On clicking submit after completing the assignment, the servlet creates an ASCII file. The filename is obtained by appending $\mathrm{R}$ to the student number supplied and adding the the (.txt) extension (e.g. R9654092.TXT). If a student fails to enter the student number, it uses a default filename "RESULTS.TXT". The file will contain the student's name, student number, the IP number or the host name of the client computer from which the answers are filled. These are stored in this file and kept in the directory c:Itomcat $\backslash$ on the server, for the lecturer to mark later. The output is given below.

Student number : 9800479

Surname : MADISHA Connecting from 220.96.21.133

$<$ ANSWERS BEGIN $>$

Question1:A

Question2: C

Question3 : (B) Pole to People Protocol

Question4 : Browser

Question5 : An organized collection of records

Question6: A

Question7 : B

Question8: A

Question9 : B

Question10: A

Question11: C

Question 12: D

Question 13: D

Question14: A

Question 15: B

Question 16: G

Question 17 : B

Question18:B 
Question19: G

Question20:B

$<$ END $>$

Figure 3. Student answer file stored on the server

On clicking submit after completing the assignment, the servlet creates an ASCII file. The output is given in Figure 3 above.

The filename is obtained by appending $\mathrm{R}$ to the student number supplied and adding the the (.txt) extension (e.g. R9654092.TXT). If a student fails to enter the student number, it uses a default filename "RESULTS.TXT". The file will contain the student's name, student number, the IP number or the host name of the client computer from which the answers are filled. These are stored in this file and kept in the directory c: Itomcat $\backslash$ on the server, for the lecturer to mark later.

The lecturer can then print the file at his own convenience and mark the answers submitted. The servlet finally calls a JSP/HTML page which acknowledges the receipt of the student's answers.

\section{Post-implementation Assessment and Results}

The whole group of students offering the 3 subjects mentioned earlier were subsequently reassessed at the end of the semester. They were given an exercise to create an email address for a friend using the South African domain "webmail.co.za'. It was observed that most of the students in the group who participated and subsequently submitted assignments via the on-line system developed, have become more proficient in the use of the Internet. The result showed dramatic improvement in the level of their knowledge and use of the Internet as well as increased speed of carrying out assigned tasks on the Internet.

\section{Conclusion}

This study concluded that the use of the online testing system have the desired effect of improving the quality of Internet knowledge of students offering introductory computer-based subjects. The online testing system also saved time, disk and papers. Students were able to do the assignment at their own time and directly to the server.

\section{Further Developments Arising from the Study}

What then? If the system led to improving the quality of Internet knowledge of students, what then can be done so it can benefit the Technikon community?

The result led to the author's idea in the participation by a team of 6 members of staff, in a project to equip an "Internet open lab", and conduct computer trainings for staff and students of the Technikon in IT applications like MsOffice and Programming (HTML, C++, Java, VB, ASP using VBScript). This is fortunately funded by USAID to the tune of R1 million (US\$147,000.00). The project was completed in August 2003, but the lab continues to serve the University community in the Soshanguve campus.

The study also had a significant impact on the IT diploma curriculum because it is an embodiment of major subjects in the NDIT curriculum namely: Internet/WWW+ HTML, Networking, JavaServlet/C++/VB, Scripting languages, Database and Project Management.

It is thus a MODEL that IT students should look towards mastering. In fact, at the beginning of the semester, the author, as the lecturer for the subject: Client-Server technology, always ask the student to connect to the web site and execute the program. The students are then charged to strive towards having such system as an outcome of the subject at the end of the semester. That gives the students a focus and a goal. This is thus an evidence of how research aids teaching. 


\section{References}

Cooper, R. (2001). Software for managing Web sites, South African Institute of Computer Scientists and Information Technologists (SAICSIT) Annual conference. Sept. 2001. Pretoria, South Africa.

Corbetta, P. (2003). Social Research: Theory, Methods and Techniques. Sage Publications, U.K. ISBN 07619-7252-8.

Magelang Institute. (1998). Servlet Tutorial. 1998. [Online] Hosted by Sun MicroSystems, Inc. Retrieved August 10, 1999. [Online] from http://developer.java.sun.com/developer/infocs/index.html

Sun MicroSystems, Inc. (1999) Java Servlet Development Kit - API Documentation. [Online] Retrieved August 10, 1999 from http://www.javasoft.com

Sun MicroSystems, Inc. (1999). JDBC Guide: Getting Started. 1997. Retrieved 10 August, [Online] Available at $\quad<\mathrm{http}: / / \mathrm{www}$.wombat.eng.sun.com/jdbc

Sun Educational services. (2002). Web Component Development With Java Technology. SL-314 Student Guide. Revision A.1. Sun Microsystems, Inc. USA.

Taylor, A. (1997). JDBC Developer's Resource. ( $2^{\text {nd }}$ ed.). Prentice Hall. USA.

\section{Biography}

Johnson Dehinbo is a senior lecturer at the Department of Computer Studies, Tshwane University of Technology, Pretoria, South Africa (recent merging of Technikon Pretoria, Technikon Northern Gauteng, and Technikon North West to form a dynamic University of Technology). $\mathrm{Mr}$ Dehinbo joined the Technikon Northern Gauteng as a lecturer in 1997. Mr Dehinbo has previously worked as a Computer Programmer / Analyst at the International Institute of Tropical Agriculture, Ibadan, Nigeria from 1991 to 1996, and as a Graduate Assistant at the Ogun State University, Ago-Iwoye, Nigeria from 1990 to 1991.

His area of research interests includes web-based application development, database, e-commerce and their impact on educational systems. 